\title{
PRESENCIA DE LA ALIANZA LINARION PEDUNCULATAE EN LOS CORDONES DUNARES GALAICO-PORTUGUESES
}

\author{
Jesús IZCO, Pablo GUITIAN \& Javier GUITIAN
}

\begin{abstract}
RESUMEN: La asociación Violo henriquesii-Silenetum littoreae comprende las comunidades terofíticas de la dunas del sector Galaico-Portugués; el sintaxon se subordina a la alianza Linarion pedunculatae. Se realiza además la validación del nombre de la alianza y de la asociación Ononido variegatae-Linarietum pedunculatae.
\end{abstract}

SUMMARY: The association Violo henriquesii-Silenetum littoreae includes the terophytic communities of the dunes from Galaico-Portuguese sector. This sintaxon is included in the alliance Linarion pedunculatae. The names Ononido variegatae-Linarietum pedunculatae and Linarion pedunculatae are valid.

Palabras clave: Vegetación, dunas, Peninsula Ibérica.

Key words: Vegetation, dunes, Iberian Peninsula.

\section{INTRODUCCION}

Galicia, con más de $1.700 \mathrm{Km}$ de costa, constituye un territorio ideal para el estudio de la vegetación litoral. Algunos aspectos parciales han sido abordados en los últimos años (Alvarez, 1972; Rivas-Martínez, 1972, etc.), pero las comunidades terofiticas de arenas móviles, a pesar de su indudable interés biogeográfico, permanecen mal conocidas.

El presente trabajo trata de dar a conocer la composición floristica y relaciones de los pastizales anuales no nitrófilos de las dunas vivas de las costas atlánticas del extremo occidental de la Península Ibérica.

LAS COMUNIDADES TEROFITICAS DUNARES EN EL OCCIDENTE PENINSULAR.

La descripción de la alianza Linarion pedunculatae por Diez Garretas, Asensi y Esteve (1975) supuso una interesante aportación a la clarificación de estas comunidades, al menos en el tramo comprendido entre el Cabo de Gata y el Cabo San Vicente. Los citados autores, además de referir la base floristica de dicha alianza, hacen referencia a la asociación ononido variegatae-Linarietum pedunculatae, tratándose en ambos 
casos de nombres inválidos, de lo que nos ocuparemos posteriormente.

Rivas-Martinez \& al. (1980) estudian los terófitos de arenas móviles de Doñana, describiendo dos nuevas comunidades, una de Linaria mumbyana var. pygmaea y otra de Ononis variegata y Silene littorea, que subordinan a la citada alianza. Con posterioridad Díez Garretas (1984) describe para las costas portuguesas, al sur del Cabo San Vicente, otra asociación que denomina Herniario algarbicae-Linarietum ficalhoanae incluible también en la alianza Linarion pedunculatae.

Para las costas cantábricas Díaz \& Navarro (1978) dan a conocer dos asociaciones en el seno de la amplia alianza Thero-Airion: Asterolino-Rumicetum y Petrorhagio-Trifolietum arvensis, aunque ya en medios dunares relativamente fijados.

\section{LAS COMUNIDADES TEROFITICAS DE LAS DUNAS GALLEGAS}

En el tramo comprendido entre las costas cantábricas y el Cabo San Vicente, a pesar de existir información sobre la vegetación costera (Be11ot, 1968; J. \& G. Braun-Blanquet, P. da Silva \& Rozeira, 1972; etc.), este tipo de comunidades no ha sido estudiado debido, probablemente, a que fueron interpretadas como integrantes de otras formaciones dunares más conspicuas. Para estos territorios es necesario, a nuestro juicio, proponer una nueva asociación que denominamos Violo henriquesii-Silenetum littoreae cuya composición florística aparece recogida en la tabla $I$. Se trata de una comunidad de

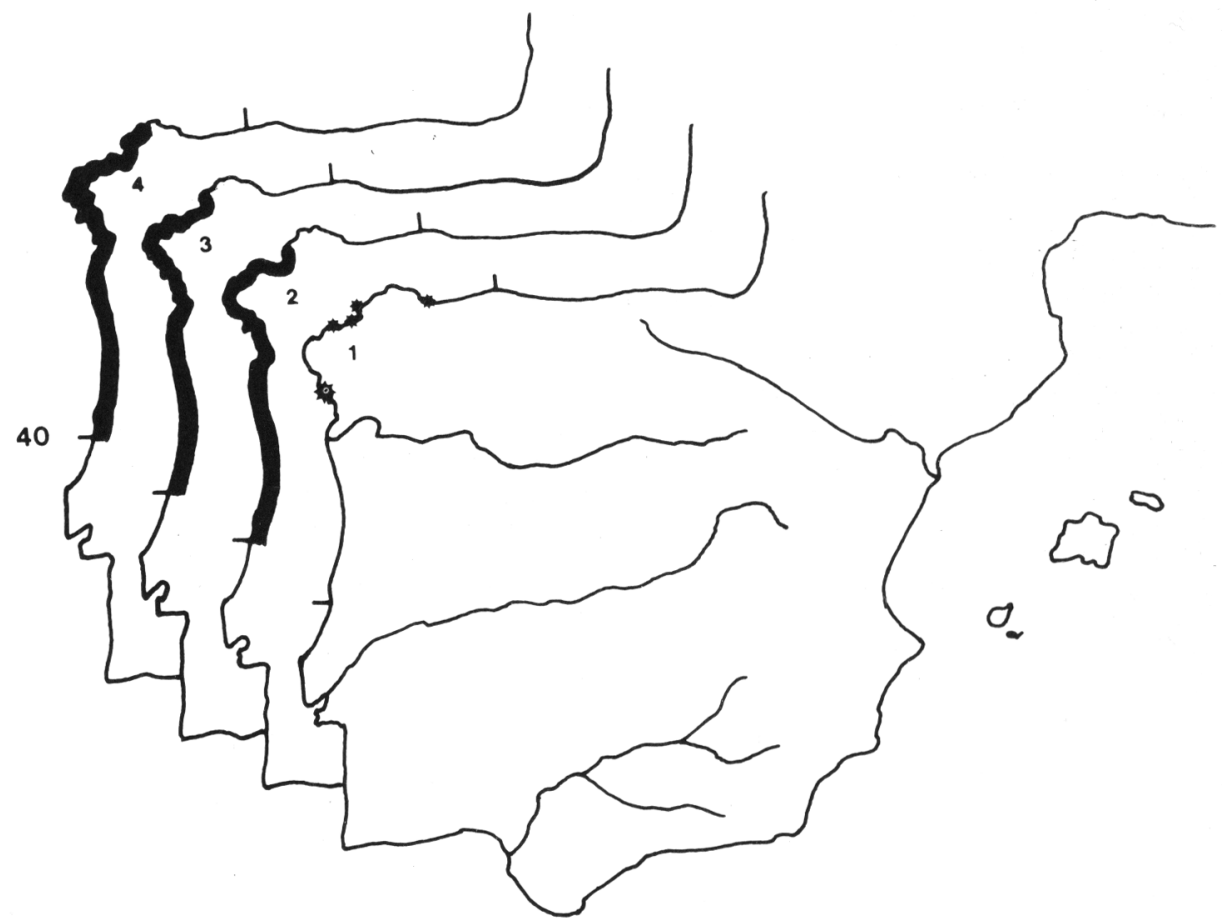

Figura 1.- Distribución en el noroeste ibérico de las características de asociación y alianza. (Se representan únicamente las distribuciones al norte del paralelo 40): 1.Omphalodes littoralis subsp. gallaecica; 2.- Viola kitaibeliana var. henriquesii; $3 .$. Silene littorea; 4.- Pseudorlaya pumila forma microcarpa. 
TABLA

Violo henriquesii-silenetum littoreae as. nova

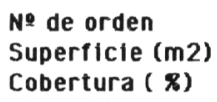

$\begin{array}{ccccccccccccc}1 & 2 & 3 & 4 & 5 & 6 & 7 & 8 & 9 & 10 & 11 & 12 & 13 \\ 4 & 5 & 2 & 2 & 4 & 5 & - & 1 & 10 & 10 & 5 & 3 & 1 \\ 30 & 20 & 30 & 20 & 25 & 15 & - & 30 & 30 & 30 & 20 & 15 & 20\end{array}$

Características de asociación y alianza (Violo henriquesii-Silenetum Iittoreae, Linarion pedunculatae)

\section{Silene littorea}

Viola kitaibeliana var. henriquesii

Psedorlaya pumlla var. microcarpa

omphalodes littoralis subsp. gallaecica $\begin{array}{lcccccccccccc}11 & 11 & + & 11 & 11 & 11 & 11 & 22 & 22 & + & 11 & \\ 11 & 11 & 11 & 11 & 11 & + & . & + & + & 22 & . & 11 & 11 \\ + & + & 22 & + & 11 & 11 & + & 22 & + & . & 11 & + & \\ + & +2 & . & + & . & . & . & 22 & . & . & . & . & 11\end{array}$

\section{Características de orden y clase}

(Malcolmietalia, Tuberarietea guttatae)

Erodium cicutarium subsp. bipinnatum

Policarpon diphyllum

Asterolinon linum-stellatum

Cerastium pumilum

Medicago littoralis

Malcolmia ramosissima

\section{Compañeras}

Cerastium diffusum

Mibora verna

Sedum arenarium

Rumex bucephalophorus subsp. hispanicus

Vulpia alopecurus

Veronica arvensis

Malcolmia littorea

Lagurus ovatus

Romulea clusiana

Paronychia argentea

Desmazeria maritima

Seneclo gallicus

Papaver rhoeas

Sonchus oleraceus

Anagallia arvensis

Ononis diffusa

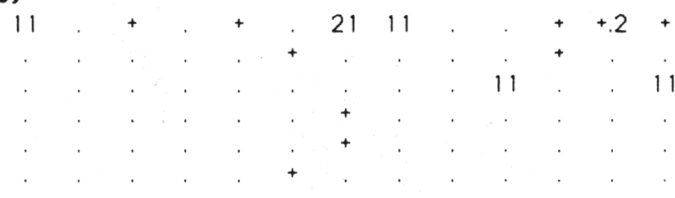
1. Playa de Louro (C) $(6 / 4 / 1984)$
2. Playa de Serans (C) $(23 / 4 / 1984)$
3. Playa de Vilar (C) $(12 / 5 / 1985)$
4. Playa de Xuño (C) $(26 / 5 / 1983)$
5. Playa de Xuño (C) $(23 / 4 / 1984)$
6. Playa de Xuño (C) $(29 / 5 / 1985)$
7. Playa de Ribeira (C) (10/4/1986)

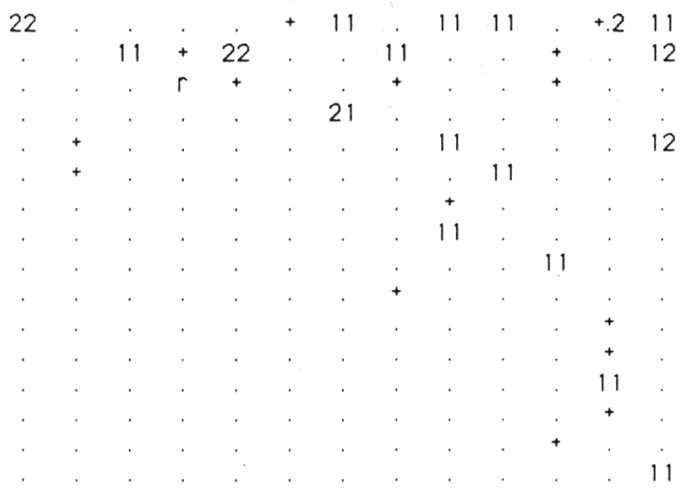

8. Playa de Vilar (C) (26/4/1984)

9. Islas Cies (PO) (4/4/1986)

10. Isla de Ons $\left(P_{0}\right)(13 / 4 / 1986)$

11. Península del Grove (Po) $(23 / 5 / 1986)$

12. Islas Cies (Po) $(10 / 4 / 1986)$

13. Playa de Dontños (C) (17/4/1985) 
desarrollo primaveral, con escasa cobertura y un número medio de 8 especies/inventario. Desde el punto de vista floristico se encuentra muy bien definida por la presencia de las caracteristicas Viola kitaibeliana var. henriquesii, Silene littorea y. Pseudorlaya. pumila forma microcarpa. La presencia del endémico Omphalodes littoralis subsp. gallaecica contribuye a definir florística y corológicamente el sintaxon.

Tipo: tabla I, invt. 8 .

Desde el punto de vista de su corología la asociación y, por consiguiente la alianza, pueden ser reconocidas en el sector Galaico-Portugués, rebasando sólo, de forma excepcional, el Golfo Artabro; los mapas de distribución de la figura 1 son en este sentido concluyentes para fijar su limite septentrional. Con la información de que disponemos parece posible su presencia en las costas portuguesas, al norte del sector Beirense litoral.

Respecto a su posición sintaxonómica, resultan evidentes sus afinidades por el orden Malcolmietalia y por la alianza Linarion pedunculatae -aunque con un cierto empobrecimiento-; apuntan en este sentido la presencia de Silene littorea, Erodium cicutarium subsp. bipinnatum, Polycarpon diphyllum y Pseudorlaya pumila forma microcarpa (tabla II). Este componente florístico tiene en nuestra opinión mayor peso que aquel que la relaciona con los Tuberarietalia, al menos a la vista de las características señaladas para estos por Rivas-Martinez (1975). Dentro del orden Malcolmietalia parece clara la imposibilidad de subordinar la asociación a alianza distinta de la citada.

otro argumento favorable a la presencia aqui de los Linarion pedunculatae es el hecho de que otras muchas comunidades procedentes de las costas atlánticas meridionales ibéricas alcanzan su limite norte el sector Galaico-Portugués; este es el caso de la alianza Helichrysion picardii o de las comunidades presididas por Corema album.

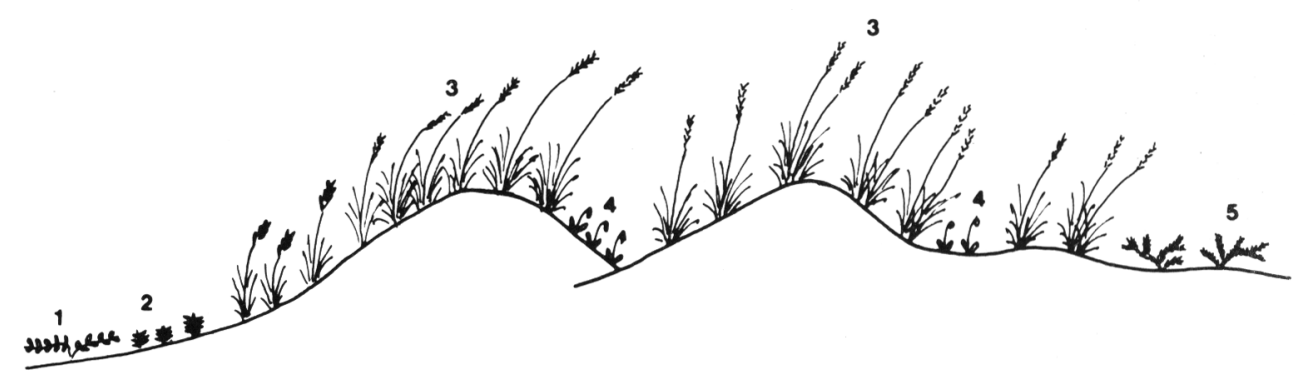

Figura 2.- Disposición de los pastizales de Violo henriquesii-Silenetum littoreae en la psamoserie: 1.- Euphorbion peplis; 2.- Euphorbio-Agropyretum junceiformis; 3.Otantho-Ammophiletum australis; 4.- Violo henriquesii-Silenetum littoreae; 5.- Seseli tortuosi-Helichrysetum picardii. 


$\begin{array}{llllll}\text { No de inventarios } & 7 & 7 & 4 & 7 & 13 \\ \text { Orden } & 1 & 2 & 3 & 4 & 5\end{array}$

Características y diferenciales de asociación

Linaria mumbyana var. pygmaea

Linaria pedunculata

Linaria ficalhoana

Herniaria algarbica

Viola kitaibeliana var. henriquesii

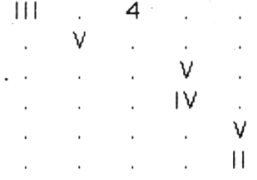

omphalodes littoralis subsp. gallaecica

\begin{tabular}{|c|c|c|c|c|}
\hline$V$ & IV & 4 & 111 & IV \\
\hline 11 & $V$ & 4 & 11 & $t$ \\
\hline & 1 & 3 & IV & IV \\
\hline 11 & $V$ & 3 & $V$ & \\
\hline & IV & 4 & $V$ & \\
\hline & 111 & 1 & 11 & \\
\hline 11 & & . & . & 1 \\
\hline & 111 & 4 & 111 & . \\
\hline 11 & & . & IV & $V$ \\
\hline 11 & & . & . & \\
\hline 11 & & . & . & . \\
\hline 1 & & . & & \\
\hline 111 & & . & . & \\
\hline & & . & 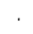 & \\
\hline & . & 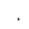 & 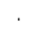 & 1 \\
\hline
\end{tabular}

\section{Compañeras}

Malcolmia littorea

Senecio gallicus

Silene colorata

Paronychia argentea

Silene nicaensis

Lotus creticus

Rumex bucephalophorus s.l.

Desmazeria maritima

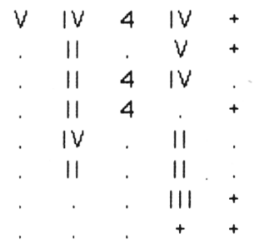

Además: Tortula arenaria. I; Trifolium tomentosum, I; Lotus castellanus, III; Leontodon taraxacoides, II y Medicago minima, I; en 1.

Aetheorhiza bulbosa, II; en 2.

Centranthus calcitrapa, 4; en 3 .

Calystegia soldanella, III; Corynephorus canescens, III; Plantago coronopus, II; Euphorbia portlandica, II; Lobularia maritima, II; Anchusa calcarea, I1; Crucianella maritima, I y Scrophularia frutescens, I; en 4 Cerastium diffusum, III; Mibora verna, III; Sedum arenarium, II; Vulpia alopecurus, 11; Asterolinon linum-stellatum, I; Veronica arvensis, I; Cerastium pumillum, +; Lagurus ovatus, +; Ononis diffusa, +; Romulea Papaver rhoeas, +; Sonchus oleraceus, + y Anagallis arvensis, +; en 5

Procedencia de las tablas: 1, Linarion pedunculatae (Rivas-Martínez \& al., 1980). 2, Ononido variegatae-Linarietum pedunculatae (Diez-Garretas, 1980). 3, Ononido-Linarietum pedunculatae subas. Iinarietosum pygmaeae (Id.). 4, Herniario algarbicae-Linarietum ficalhoanae (Id.). 5, Violo henriquesii-Silenetum littoreae as. nowa 
La posición de la asociación dentro del complejo dunar es clara. Por 10 general se inserta entre las comunidades de barrón en la duna primaria (otantho-Ammophiletum australis), más en su cara interna que en la orientada al mar; es frecuente también en los sitios donde la erosión, de uno u otro origen, ha abierto brecha en las arenas de las dunas grises posteriores colonizadas por el matorral de Seseli tortuosi-Crucianelletum.

VALIDACION DE LOS NOMBRES Linarion pedunculatae Y Ononido variegatae-Linarietum pedunculatae.

Por causas ajenas a los autores del trabajo original sobre 1.a alianza Linarion pedunculatae (Diez Garretas \& a1., 1978) no fue publicada entonces 1 a tabla que sostenia la propuesta de la asociación Ononido variegatae-Linarietum pedunculatae que, a su vez, era el tipo que sustentaba la alianza.

Para solventar esta ausencia, dichos autores repartieron con posterioridad y restringidamente una copia de la tabla. Ello no parece suficiente en el sentido de los artículos 1 y 2 a de CNF para que podamos considerar válida la publicación del nombre puesto que no fue remitida a una porción considerable de fitosociólogos, ni a bibliotecas públicas, ni siquiera a todos los suscriptores de "Documents Phytosociologiques", donde se publicó la asociación.

La referencia posterior de Díez Garretas (1984) no resuelve el problema nomenclatural. Si bien publica una tabla de la Ononido variegatae-Linarietum pedunculatae, resulta una propuesta inválida por falta de tipo nomenclatural (Art. 5); 1a nueva Ononido-Linarietum subas. Linarietosum pygmaeae tampoco está válidamente publicada a pesar de la mención de un tipo nomenclatural. La invalidez de la propuesta radica en la publicación de una subasociación subordinada a una asociación inválidamente publicada (Art. 4).

En el mismo trabajo (1.c.) se propone una segunda asociación publicada válidamente bajo el nombre Herniario algarbicae-Linarietum ficalhoanae. Esta única asociación valida el nombre de la alianza Linarion pedunculatae - a la que se subordina de forma clara e inequívoca- y es su tipo nomenclatural (Arts. 5 y 8 ) *.

Con objeto de subsanar los defectos que impidieron la publicación válida del nombre Ononido variegatae-Linarietum pedunculatae con anterioridad, ofrecemos a su autor el proceder aqui a fijar el tipo y proponer el sintaxon:

as. Ononido variegatae-Linarietum pedunculatae Díez Garretas nova

subas. linarietosum pedunculatae (Tipo: Armaçao da Pera, Portugal. area: 2m2. Cobertura: 40\%).

Caracteristicas de asociación y unidades superiores:

$\begin{array}{lrcr}\text { Pseudorlaya pumila } & + & \text { Polycarpon alsinifolium } & + \\ \text { Ononis variegata } & 2.2 & \text { Cutandia maritima } & 1.1 \\ \text { Linaria pedunculata } & + & \text { Compañeras } & \\ \text { Erodium cicutarium } & & \text { Malcolmia littorea } & 1.1 \\ \text { subsp. bipinnatum } & + & \text { Silene nicaensis } & 1.1\end{array}$

(*) De forma análoga hemos procedido con anterioridad (Izco, Molina E Fernández-Gonzáleź (1986) e Izco (1988). 
subas. linarietosum pygmeae Díez Garretas nova (Tipo: Meia Praia, Lagos, Portugal. area: $2 \mathrm{~m} 2$. Cobertura: $40 \%$.

Características de asociación y unidades superiores:

$\begin{array}{lccr}\text { Pseudorlaya pumila } & + & \text { Cutandia maritima } & 1.1 \\ \text { Linaria mumbyana } & & \text { Compañeras } \\ \quad \text { var. pygmaea } & 1.1 & \text { Silene colorata } & \\ \text { Medicago litoralis } & +.2 & \text { subsp. pubicalycina } & 1.1 \\ \text { Polycarpon alsinifolium } & 1.1 & \text { Paronichia argentea } & + \\ \text { Erodium cicutarium } & & \text { Malcolmia littorea } & + \\ \text { subsp. bipinnatum } & + & \text { Centranthus calcitrapa } & +\end{array}$

Ambos inventarios tipo proceden de la tabla 4 de Díez Garretas (1984), no 5 y 10 respectivamente.

En consecuencia, el esquema sintaxonómico queda como sigue:

A1. Linarion pedunculatae Diez Garretas 1984

As. Herniario algarbicae-Linarietum ficalhoanae Díez Garretas 1984 (Tipo de la alianza)

As. Ononido variegatae-Linarietum pedunculatae Diez Garretas as. nova in Izco, P. et J. Guitian subas. typicum

subas. 1inarietosum pygmaeae Diez Garretas nova in Izco, P. et J. Guitian

As. Violo henriquesii-Silenetum littoreae Izco, P. et J. Guitian nova

NOTA: Los taxones que se mencionan tanto en el texto como en las tablas están en concordancia nomenclatural con Flora Europaea (Tutin \& al. (ed.) 1964-1980) salvo en los casos que se mencionan a continuación: Linaria mumbyana Boiss. \& Reuter var. pygmaea (Sampaio) Sampaio; Omphalodes littoralis Lehm. subsp. gallaecica Láinz; Pseudorlaya pumila (L.) Grande forma microcarpa (Loret \& Barrandon) Sáenz; Viola kitaibeliana Schultes var. henriquesii (Willk.) Beck.

\title{
AGRADECIMIENTOS
}

Agradecemos a la Dra. Fernández Casado la revisión del material del género Viola.

\author{
BIBLIOGRAFIA
}

ALVAREZ, R. -1972- Estudio de la Flora y la Vegetación de las playas de Galicia. Trab. Compost. Biol., 2:35-65.

BARKMAN, J., MORAVEC, J. \& RAUSCHERT, S. -1986- Code of Phytosociological Nomenclature. Vegetatio, 67(3):145-195.

BRAUN-BLANQUET, J., BRAUN-BLANQUET, G., ROZEIRA, A. \& PINTO DE SILVA, A. -1972- Resultats de trois excursions géobotaniques à travers le Portugal septentrional et moyen. IV. Essquise sur la végetation dunale. Agronomia Lusitana, 33:217-234. 
DIAZ, T.E.\& NAVARRO, F. -1978- Las comunidades de Thero-Airion R. Tx. 1951 en las playas asturianas: su posición fitotopográfica. Anal. Inst. Bot. Cavanilles, 34(2).:571-596.

DIEZ GARRETAS, B. -1984- Datos sobre la vegetación psammófila de las costas portuguesas. Doc. Phytosoc. N.S., 8:71-81.

DIEZ GARRETAS, B., ASENSI, A. \& ESTEVE, F. -1978- Pastizales terofíticos de las playas y dunas del sur de la Península Ibérica. Colloq. Phytosoc., $6: 73-79$.

IZCO, J. -1988- Problems of Phytosociological nomenclature: author citation following automatic validation of the name an alliance (en prensa).

IZCO, J., MOLINA, A. \& FERNANDEZ-GONZALEZ, F. -1986- Pastizales nanoterofíticos mediterráneos: Thero-Brachypodion y Sedo-Ctenopsion. II. Eco. Medit., 12(3-4):89-103.

RIVAS-MARTINEZ, S. -1972- Vegetatio Hispaniae, Notula III. Bol. Real Soc. Esp. Hist. Nat. (Biol.), 70:153-162.

RIVAS-MARTINEZ, S. -1977- Sur la sintaxonomie des pelouses therophytiques de l'Europe Occidentale. Colloq. Phytosoc., 6:55-71.

RIVAS-MARTINEZ, S., COSTA, M. CASTROVIEJO,S. \& VALDES, E. -1980- Vegetación de Doñana (Huelva, España). Lazaroa, 2:5-189.

SAENZ DE RIVAS, C. -1975- Datos sobre el género Pseudorlaya (Murb.) Murb. (Umbelliferae). Anal. Inst. Bot. Cavanilles, 31(2):191-204. 